\title{
Infléchir le destin car la vraie souffrance est à venir
}

(Société maure-islam sunnite)

\section{Corinne Fortier}

\section{(2) OpenEdition Journals}

Édition électronique

URL : https://journals.openedition.org/span/736

DOI : $10.4000 /$ span.736

ISSN : 2268-1558

Éditeur

École pratique des hautes études. Sciences humaines

Édition imprimée

Date de publication : 1 juin 2005

Pagination : 195-217

ISSN : 0294-7080

Référence électronique

Corinne Fortier, «Infléchir le destin car la vraie souffrance est à venir », Systèmes de pensée en Afrique noire [En ligne], 17 | 2005, mis en ligne le 06 juin 2013, consulté le 21 septembre 2021. URL : http:// journals.openedition.org/span/736; DOI : https://doi.org/10.4000/span.736 


\section{Infléchir le destin car la vraie souffrance est à venir (société maure-islam sunnite)}

\section{Corinne Fortier}

Chargée de Recherche au CNRS Laboratoire d'Anthropologie Sociale (CNRS-EHESS-Collège de France)

\footnotetext{
"Ce Jour-là, vous serez exposés en pleine lumière; aucun de vos secrets ne restera caché " (Coran, trad. Masson, 1967, t. 2 : 714).

"Le châtiment dans la tombe est une réalité " (Hadith, Bukhârî, 1964 : 92, § 13).
}

On ne peut comprendre la vie quotidienne des Musulmans chez lesquels la foi islamique est profondément enracinée - comme c'est le cas des Maures ${ }^{1}$ de Mauritanie - sans la relier à cette autre vie qu'ils connaîtront dans l'audelà (âkhira), qui est pour eux aussi réelle que celle qu'ils vivent ici-bas (dunya). Mais ce fait, relevant de la foi individuelle plus que du rituel collectif, de l'éthique religieuse plus que du comportement social, est difficilement saisissable par les sciences sociales.

Il est assez rare, en effet, que les anthropologues travaillant dans des sociétés musulmanes s'intéressent aux représentations eschatologiques islamiques et à leur impact sur la vie des individus. Cela s'explique par plusieurs raisons. D'une part, cette question demandant de se tourner vers l'islam et ses textes, ils l'ont considérée comme ne relevant pas de leur domaine, mais plutôt de l'orientalisme. D'autre part, une telle recherche, touchant non seulement aux représentations de l'au-delà mais aussi à la manière dont elles déterminent les conduites individuelles, se révèle difficile à mener puisqu'elle concerne les motivations des

\footnotetext{
${ }^{1}$ La société maure, comme la plupart des sociétés d'Afrique du Nord, est islamisée selon le rite malékite d'obédience sunnite depuis très longtemps, à l'origine par les Almo. ravides au $\mathrm{Xl}$ e siècle. Les Maures, qui parlent un dialecte arabe, le hassâniyya, restent culturellement des bédouins même s'ils ne nomadisent quasiment plus.
} 
${ }^{2}$ Nous avons traité par ailleurs de la difficulté des occiden. taux, héritiers de la culture chrétienne, à percevoir la dimension spirituelle de l'islam derrière ce qu'ils jugent « prosaïque » (Fortier, 2003). individus, qui restent souvent inconnaissables. De plus, les intéressés eux-mêmes parlent peu de cette question; dans la mesure où elle regarde le croyant dans son rapport à Dieu, elle appartient à la foi intime, qui ne s'exhibe pas. Enfin, la dimension du salut étant fondamentalement religieuse, elle a été négligée par un type d'explication qui cherche les raisons d'agir plutôt du côté de l'organisation socio-culturelle alors même que certaines pratiques sociales sont le fruit d'une démarche individuelle d'ordre religieux.

Par ailleurs, c'est non seulement la conduite des Maures, en tant que musulmans, qui est influencée par leur représentation de l'au-delà mais également leurs affects. Ainsi, la manière dont ils accueillent la dimension du malheur dans leur vie, et en particulier un deuil, est certes liée à leur soumission résignée devant la volonté divine mais dérive aussi de la croyance selon laquelle cette souffrance est toute relative par rapport à celle qui les attend après leur décès.

Les morts, en islam, sont en effet particulièrement malmenés à différentes étapes de leur destin posthume, situation que les Maures redoutent pour eux-mêmes et pour leurs proches; tout d'abord, lors de l'interrogatoire terrifiant imposé par les anges dès l'inhumation, puis, dans un deuxième temps, par les châtiments physiques que les morts pourront recevoir dans leur tombe en punition de leurs mauvaises actions, ensuite, par le cataclysme que représentera pour chacun le jour du Jugement dernier, et enfin par les tourments de l'enfer que beaucoup seront amenés à connaître.

Aussi, bien qu'une certaine vision occidentale de l'islam tende à se focaliser sur le paradis musulman dans la mesure où il promet des bienfaits physiques qu'une morale chrétienne juge peu spirituels ${ }^{2}$ et en dépit du fait que, du point vue de l'islam, tous les fidèles aient un jour à connaître le paradis, c'est la dimension terrifiante de ce qui attend les Maures après leur mort - entre autres, la période plus ou moins longue qu'ils pourront éventuellement passer en enfer - qui les préoccupe prioritairement et oriente de façon invisible nombre de leurs comportements. 


\section{Exprimer pieusement sa peine}

Quoique les émotions ressenties au décès d'un proche relèvent du domaine individuel, leur expression est plus ou moins codifiée socialement et peut prendre des formes diverses allant de l'intériorisation individuelle à la représentation spectaculaire. Dans la société maure, il n'existe pas, comme dans d'autres sociétés du Maghreb $^{3}$ et de Méditerranée ${ }^{4}$, de pleureuses professionnelles, et on évite même de manifester son émotion à la mort d'un proche, attitude qui paraît directement inspirée des sources scripturaires sunnites fondamentales ${ }^{5}$.

Dans l'islam sunnite, la conduite à tenir en cas de deuil dérive de celle du Prophète ${ }^{6}$. Mahomet aurait déclaré à ce sujet : "Il est deux choses qui sont répandues chez les gens et qui sont de la mécréance $(k u f r)$ : mettre en doute la généalogie de quelqu'un et les gémissements outranciers pour pleurer les morts " (Nawawy, 1991 : 415).

Le fait que les lamentations soient assimilées dans ce hadith à la mécréance s'explique en ce que ce type de conduite était caractéristique des mœurs sémitiques de l'époque. Ainsi, on peut avancer l'hypothèse qu'en condamnant un tel comportement, l'islam tient à se distinguer nettement des habitudes des anciens Arabes et des Juifs?

$\mathrm{Si}$, dans la société maure et en islam sunnite, l'attitude des vivants vis-à-vis du mort peut avoir des conséquences positives sur son salut (Fortier, 2005), elle peut aussi avoir des effets négatifs comme le montre le fait que les lamentations nuisent aux défunts; plusieurs hadith en témoignent : "Le mort sera châtié à cause des pleurs des siens " (Bûkhârî, 1993 : 258, § 654) et «Les pleurs des vivants sur le mort sont pour lui un supplice " (bukâ'al-hayyi al-mayyiti adhâbun li-l-mayyiti) (Wensinck et Mensing, 1967, t. $6: 297)^{8}$.

Par ailleurs, l'islam sunnite distingue les lamentations des pleurs sincères. La conduite du Prophète est encore à la source de cette distinction, celui-ci n'ayant pu contenir son émotion à la mort de l'un de ses fils : «Ibrahim, à l'âge de dix-sept mois mourut, sous les yeux de son père qui ne put

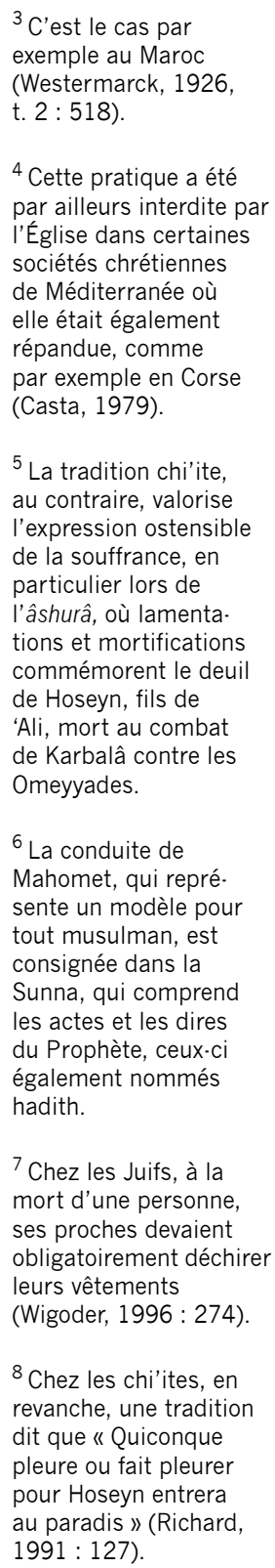

${ }^{7}$ Chez les Juifs, à la mort d'une personne, ses proches devaient obligatoirement déchirer leurs vêtements (Wigoder, 1996 : 274).

${ }^{8}$ Chez les chi'ites, en revanche, une tradition dit que « Quiconque pleure ou fait pleurer pour Hoseyn entrera au paradis » (Richard, 1991 : 127). 
${ }^{9}$ Cette attitude résignée n'est cependant pas toujours la règle dans les pays musulmans, par exemple dans certaines sociétés du Maghreb où il existe des pleureuses profession nelles, ou encore en Égypte où hommes et femmes du cortège funéraire poussent des cris et des sortes de lamentations (Galal, $1937: 172)$ retenir un torrent de larmes. Ce que voyant et se rappelant que le Prophète, en cas de deuil, avait interdit les lamentations et défendu de se déchirer le visage et les vêtements, $\mathrm{Abd}$ al-Rahman ben Aouf lui dit : "Toi aussi, Ô envoyé d'Allah." "Ô Ben Aouf, lui répondit-il, les larmes sont en effet de la compassion, et elles ne sont pas interdites comme les cris et les lamentations, qui sont des protestations inspirées par le démon contre les décrets de la providence." Puis, ses larmes recommençant à jaillir avec plus d'abondance encore, il ajouta: "Les yeux pleurent, le cœur est affligé, mais nous ne proférons aucune exclamation déplaisante au Seigneur. C'est au premier choc que la véritable résignation se révèle, car, plus tard, elle a le secours du temps. Ô Ibrahim, nous sommes dans une profonde tristesse causée par ta séparation; mais nous appartenons à Allah, et à Lui nous devons retourner" "(Dinet et Ben Ibrahim, s.d. : 229).

Dans ce hadith, les lamentations sont condamnées dans la mesure où elles apparaissent comme l'expression d'une révolte contre la volonté divine. Par conséquent, plus encore que pour se démarquer des usages sociaux ou religieux antérieurs, l'islam condamne les lamentations en tant qu'elles témoignent de la non-acceptation de la puissance de Dieu dont un des pouvoirs suprêmes est de donner la vie et la mort.

\section{Accepter la volonté de Dieu}

En cas de deuil, dans la société maure, l'attitude résignée des proches du défunt ${ }^{9}$, qui témoigne de leur acceptation de la fatalité (ridha bi-l-qadhâ'), est encouragée chez ceux qui sont capables de surmonter leur peine; une telle attitude est préconisée par le droit malékite : « Il ne sied pas de répandre des larmes à ce moment. Mais une patience pleine de dignité et une noble résignation sont plus méritoires pour qui a assez de force d'âme pour cela » (Qayrawânî, s.d. : 105).

Ainsi, le croyant est appelé dans ces circonstances à la patience (sabr) devant l'épreuve, vertu considérée, en islam, 
comme cardinale (makârim al-akhlâq). Une des formules de condoléances utilisée par les Maures fait allusion à cette vertu de constance dans l'adversité : «Que Dieu vous donne la patience de supporter cela » (Allah yasbarkum 'âlih). Quant aux proches du défunt, résignés face à cet événement irrémédiable, ils répètent inlassablement : «Telle est la décision divine " (qadrat mulâna) et rendent par ailleurs grâce à Dieu que leur infortune ne soit pas plus grande en proclamant : "Louange à Dieu, par lequel cela n'est pas plus grave "(hamdulilah illi mahu a'zam).

Lorsqu'un événement dramatique, comme un accident, provoque la disparition de plusieurs êtres chers, reste la présence consolatrice de Dieu, ainsi que l'indique cette formule religieuse utilisée dans la société maure: "Nous nous en remettons à Dieu, il est notre meilleur garant " (hasbunâ Allah wa nima al-wakîl) (Sharaf, 1987 : 66). Dieu est "le vivant-subsistant-par-soi " (hay al-qayyum) dont la présence est éternelle; il représente un point fixe inébranlable pour les hommes, qui ne sont que de passage dans ce monde.

Dans la société maure, l'attitude résignée qui sied aux proches s'étend également à celui qui meurt. Cette digne acceptation de la mort au moment fatidique se manifeste aussi bien lorsque l'individu décède de façon naturelle ou accidentelle. C'est ce que montre le comportement d'un berger de Chinguetti qui mourut de soif après s'être égaré dans le désert à la recherche de ses chameaux ${ }^{10}$. Il avait utilisé ses dernières forces pour creuser sa tombe dans le sable. Puis, il s'y était étendu selon la position religieuse recommandée, attendant pieusement que la mort vienne le prendre. Auparavant, afin d'être en état de pureté, il avait enlevé son vêtement; ainsi, il avait été retrouvé mort et enterré, son sarwâl à ses côtés.

L'islam étant soumission à la volonté divine, si Dieu a choisi de reprendre la vie qu'il a créée, le fidèle ne peut que s'en remettre à sa décision comme le rappelle la formule coranique (II, 156) prononcée à l'occasion d'un décès : "Nous sommes à Dieu et à lui nous retournons " (innâ lillâhi wa innâ ilayhi râji îun).

${ }^{10}$ Cet événement a eu lieu lors de l'un de nos séjours à Chinguetti. 
${ }^{11}$ Selon l'embryogenèse islamique, c'est le quatrième mois, ou plus exactement le cent vingtième jour, que Dieu envoie un ange qui détermine une durée de vie et insuffle l'âme (rûh) à ce qui n'est encore qu'une boule de chair (mudgha), l'ange obéissant alors à l'ordre divin : «Écris ses œuvres, sa part de la vie, son terme, et sa destinée heureuse ou malheureuse » (Bukhârî, 1993, t. $2: 556$, $\S 1356)$. Nous avons développé ce sujet dans un précédent article (Fortier, 2001).
Le mot même qui désigne la mort en arabe, wafât, renvoie au terme et à l'accomplissement, la mort réalisant la fin du cycle de vie d'un être. Ce cycle, prédéterminé par Dieu au moment où l'individu est encore dans le ventre de sa mère ${ }^{11}$, ne fait alors que s'actualiser lorsque cette durée arrive à son terme.

Aussi, un homme ne peut-il se donner la mort sans être sévèrement châtié dans l'au-delà pour avoir rivalisé avec les desseins de Dieu; comme le déclare un hadith : "Celui qui se suicide sera supplicié le jour du Jugement avec l'instrument de son suicide " (Nawawy, 1991 : 406). À cet égard, nous n'avons jamais entendu parlé de cas de suicide dans la société maure.

\section{Vivre ici et maintenant en pensant à l'au-delà}

Les Maures se préparent à l'instant de leur mort qui viendra tôt ou tard; comme le suggère un proverbe arabe ancien connu en Mauritanie : "Le temps du malheur ne prévient pas " (az-zar yûlad blâ dra). Ainsi, loin de fuir tout signe qui leur rappellerait leur propre mort, ils conservent précieusement dans une malle le linceul, acquis de leur vivant, qui les recouvrira le jour des funérailles. Car la mort signifie certes la fin de la vie terrestre mais débouche également sur une autre vie, non moins importante; un hadith suggère même sa précellence : "Ô Dieu, il n'y a d'autre vie que celle de l'Autre Monde »(Bukhârî, 1964 : 87, § 2).

La présence de l'homme dans ce monde étant éphémère, il lui est conseillé de ne pas trop s'y attacher; aussi la parole pleine de sagesse du Prophète connue en Mauritanie : "Sois dans ce bas monde comme un étranger ou un passant " est-elle relayée par celle de l'un de ses compagnons, Ibn 'Umar, : "Quand tu es au soir n'attends pas le matin, et quand tu es au matin, n'attends pas le soir. Prends ta santé pour ta maladie, et ta vie pour ta mort " (Bukhârî, 1993 : 876, § 2092).

Cette pratique du détachement participe d'une éthique de l'instant qui libère des regrets du passé comme de la crainte de l'avenir; il faut savoir vivre au présent car per- 
sonne hormis Dieu ne sait de quoi demain sera fait. Cette morale semble remonter à l'Arabie préislamique puisqu'on la retrouve dans ce vers non dépourvu d'épicurisme du grand poète Imru' al-Qays (m. 550) : "Aujourd'hui le vin, et demain autre chose" (alyawma khamr/wa ghadân amr).

L'incertitude de l'avenir est en soi une invitation à profiter pleinement du présent. Tel est aussi le message porté dans tout le monde arabe par la chanteuse égyptienne Umm Kulthum (m. 1977) lorsqu'elle déclame un vers en arabe classique du poète persan 'Umar al-Khayâm (m. 1123) : « Demain m'est encore inaccessible, aujourd'hui m'appartient " (ghadân bi zahri al-ghaybi / wa al-yawma li).

Une expression maure invite par ailleurs à prendre la vie comme elle vient (bâsh jât ghazât). Tout événement mérite d'être vécu, y compris un malheur, celui-ci pouvant se révéler bénéfique. De la même façon, il arrive qu'un événement heureux s'avère funeste selon la conception métaphysique musulmane qui distingue l'apparence (zahr) de l'essence (batn).

Étant donné que l'homme se situe dans un certain aveuglement vis-à-vis du cours de sa vie, mieux vaut qu'il accueille ce qui lui arrive avec relativisme; le Coran (II, 216) avertit ainsi le croyant : «Il est possible que vous ayez de l'aversion pour une chose qui est un bien pour vous et il est possible que vous aimiez une chose qui est un mal pour vous. Allah sait, alors que vous ne savez pas " (trad. Blachère, $1980: 60$ ).

De plus, comme le déclare explicitement un verset coranique (LIII, 42-44), Dieu donne la vie et la mort, de la même façon qu'il prodigue les joies et les peines : "Qu'à ton Seigneur tout revient, que c'est Lui qui fait rire et qui fait pleurer, que c'est Lui qui fait vivre et qui fait mourir" (trad. Blachère, 1980 : 562).

Dans la mesure où tout ce qui advient en ce monde, bonheur ou malheur, est voulu par Dieu, un proverbe arabe connu dans la société maure incite à ne pas maudire la vie : "N'insultez pas la vie, car la vie c'est Dieu » (lâ tasubbû dahra inna dahra huwwa Allah). Or, ce proverbe, après comparai- 
12 L'image du feu infernal se retrouve dans de nombreuses pensées religieuses; comme le remarque J. Le Goff (1999 : 797) : « Ce qui frappe dans les doctrines et les images de l'au-delà c'est l'om. niprésence du feu. »

${ }^{13}$ Ainsi, lors d'un de nos terrains à Chin. guetti, une femme qui souhaitait pour notre bien que nous nous convertissions à l'islam, nous dit, lorsque nous exprimions notre difficulté à saisir avec la main le couscous brûlant qu'elle avait préparé, que cette sensation n'était rien en comparaison de celle qui nous attendait en enfer si nous restions infidèle. son avec des traditions prophétiques, se révèle inspiré du hadith : "Dieu a dit : "Les hommes insultent le sort. Or le sort c'est Moi, Qui tiens en Mon pouvoir le jour et la nuit" " (Bukhârî, 1964 : 225, § 2).

\section{La crainte de l'enfer et du Jugement dernier}

De nombreux Maures vivent dans la crainte d'aller en enfer (jahannam) et dans la peur de connaître les tourments de ses flammes. Ce qui caractérise en effet l'enfer c'est son feu $(n \hat{a} r)^{12}$, dont la chaleur est d'une plus grande intensité que celui qu'on peut produire ici-bas ; plus précisément, comme l'indique un hadith (Bukhârî, 1964 : 97, § 31), « le Feu de l'Enfer a de plus que le feu terrestre soixante-neuf parties dont chacune a l'ardeur du feu terrestre $»^{13}$.

Les peines, même les plus légères, qui attendent celui qui va en enfer, sont d'une grande violence, ainsi que l'atteste un hadith (Bukhârî, 1993, t. 2: 888, § 2130) : «An-Nou'man Ben Bachir a rapporté qu'il a entendu le Prophète dire : "Parmi les réprouvés de l'Enfer qui subira le châtiment le moins douloureux, au jour de la résurrection, sera un homme, on lui placera sur la plante du pied deux braises si ardentes qui feront bouillir sa cervelle"... "

C'est aussi avec tremblement que les Maures pensent au jour du Jugement dernier, et j'ai pu observer que les plus sensibles d'entre eux, comme les élèves coraniques, pleurent parfois de peur lorsqu'ils récitent les passages catastrophiques du Coran qui y font référence. Ce Livre (XXII, 2) donne en effet une description terrifiante du Jugement dernier, jour où le lien qui apparaît comme le plus indéfectible, celui qui existe entre une mère et son nourrisson, se trouvera rompu : «Le tremblement de terre de l'Heure sera sûrement quelque chose de terrible! Le jour où vous le verrez : toute femme qui allaite oubliera son nourrisson; toute femme enceinte avortera. Tu verras des hommes ivres alors qu'ils ne le seront pas. - Le châtiment de Dieu sera très dur. " 
Ce jour est celui où seront jugées les actions manifestes de l'homme mais également ses intentions les plus secrètes. Dans ce face-à-face avec Dieu, l'âme de l'individu sera à nu, car le créateur connaît le for intérieur de sa créature, ainsi que le montre un passage du Coran (LXXXII, 1-16) : "Lorsque le ciel se rompra et que les étoiles seront dispersées; lorsque les mers franchiront leurs limites et que les sépulcres seront bouleversés : toute âme saura ce qu'elle a fait de bien et de mal. Ô toi, l'homme! Comment donc as-tu été trompé au sujet de ton noble Seigneur qui t'a créé puis modelé et constitué harmonieusement; - car il t'a composé dans la forme qu'il a voulue - Bien au contraire! Vous traitez de mensonge le Jugement, alors que des gardiens veillent sur vous : de nobles scribes qui savent ce que vous faites ${ }^{14}$. Oui, les hommes bons seront plongés dans les délices et les libertins dans une fournaise où ils tomberont le Jour du Jugement sans pouvoir y échapper » (trad. Masson, 1967, t. 2 : 744).

Cependant, tous les Musulmans goûteront un jour au repos voluptueux du paradis. Certaines catégories de personnes y entreront plus rapidement que d'autres. C'est le cas, d'une part, des martyrs islamiques tombés dans un juste combat (jihâd), qui échapperont aux affres de l'enfer et n'auront pas à rendre compte de leurs actes au jour du Jugement dernier; le Coran (III, 163) en parle en ces termes : "Et ne crois point que sont morts ceux qui ont été tués dans le Chemin d'Allah! Au contraire! ils sont vivants auprès de leur Seigneur, pourvus de leur attribution " (trad. Blachère, 1980 : 99).

D'autre part, l'islam semblant considérer qu'il n'est rien de plus funeste pour des parents que de perdre plusieurs de leurs enfants, les tourments de l'enfer seront épargnés à ceux qui ont vécu cette épreuve; comme le déclare un hadith (Bukhârî, 1977 : 404-405) : « Il n'est pas un seul musulman, à qui la mort aura enlevé trois enfants n'ayant pas atteint l'âge de pécher, que Dieu ne fasse entrer dans le Paradis par suite de son extrême miséricorde envers les musulmans."

\footnotetext{
${ }^{14}$ Durant la vie de l'individu, deux anges, I'un placé sur son épaule droite et l'autre sur son épaule gauche, sont censés inscrire, le premier, ses bonnes, et le second, ses mauvaises actions.
} 
15 Dans le mazdéisme de l'Iran préislamique, on trouve également la conception d'un enfer temporaire (Molé, 1961 : 145). Chez les penseurs chrétiens antérieurs à Saint Augustin, tel Origène (m. 253.254), le paradis est aussi gagné après une période plus ou moins longue passée en enfer (Le Goff, 1999 : 858).

${ }^{16}$ En islam, seuls les courants khârijite et mu'tazilite pensent que quiconque entre en enfer y restera éternel lement (takhlîd fî-l-nâr) (Laoust, 1977 : 446).

${ }^{17}$ Le terme de « barrière » traduit ici le mot barzakh qui se retrouve à deux autres reprises dans le Coran (LV, 20 et XXV, 53). Selon le lexicographe arabe Ibn Manz̧ûr (s.d., t. $3: 8, \S 29$ ), ce terme désigne la séparation et également le délai accordé. C'est cette double acception, à la fois physique et tempo. relle du terme, qui est retenue en islam où le barzakh renvoie d'une part à la barrière infran. chissable qui sépare le mort du monde de la vie, et d'autre part au temps qui sépare le décès de l'instant de la résurrection. Selon $B$. Carra de Vaux (1975: 1104), des exégètes considèrent par ailleurs le barzakh comme une barrière entre l'enfer et le paradis. Ce terme est d'origine persane où, là aussi, il ferait référence à l'intervalle de temps entre la mort et la résurrection.
Néanmoins, la plupart des Musulmans passeront une plus ou moins longue période en enfer avant d'entrer au paradis ${ }^{15}$. Seuls les infidèles, à qui Dieu a interdit ce lieu de félicité (Bukhârî, 1964 : 97, § 30) resteront en enfer éternellement, les croyants y séjournant selon une durée variable ${ }^{16}$. Aussi mieux vaut-il, pensent les Maures, se conduire pieusement dans cette vie pour espérer échapper aux tourments prolongés de l'enfer.

Un hadith connu dans la société maure fait ainsi parler le défunt, selon qu'il a été pieux ou non, au moment où il quitte la demeure des vivants pour celle des morts : "Quand le cercueil est posé à terre et que les hommes le placent sur leurs épaules, le défunt s'il était pieux s'écrie : "Hâtez-vous de m'emporter!" Mais s'il s'agissait d'un impie, il hurle à l'intention de sa famille: "Malheur à moi, où m'emportez-vous?" " (Nawawy, 1991 : 262).

Mais une fois passé de vie à trépas, il est trop tard pour se repentir de ses mauvaises actions; ainsi que l'indique le Coran (XXIII, 99-100) : «Lorsque la mort approche de l'un d'eux, il dit: "Mon Seigneur! Qu'on me renvoie sur la terre, peut-être, alors, accomplirais-je une ouvre bonne parmi les choses que j'ai délaissées." Non !... C'est là, seulement, une parole qu'il a prononcée; une barrière ${ }^{17}$ se trouve derrière les hommes jusqu'au Jour où ils seront ressuscités » (trad. Masson, 1967, t. 2 : 427-428).

\section{CEuvrer envers autrui et envers Dieu}

Le statut des œuvres reste à définir en islam, dont la pensée oscille entre liberté et prédestination, notions qui sont articulées comme suit dans un hadith : "Agissez, et alors tout vous sera facilité pour ce en vue de quoi vous avez été créé; ceux qui seront destinés à la Félicité, on leur facilitera les œuvres des gens destinés à la Félicité; et ceux qui sont destinés au Malheur, on leur facilitera les œuvres des gens destinés au Malheur »(Bukhârî, 1964 : 87-88, § 4).

Certaines paroles du Prophète affirment en effet que le destin (maktûb), scellé pour chacun avant sa naissance, 
prévaut sur les œuvres : «Par Allâh, en dehors de Qui il n'est pas d'autres divinité, chacun de vous aurait beau œuvrer comme l'ont fait ceux destinés au Paradis, en sorte qu'il s'en approcherait à la distance d'une coudée, alors ce qui a été écrit pour lui prévaudrait, et donc il accomplirait (quand même) les actions des damnés, et il entrerait en Enfer. Et certes, chacun de vous aurait beau œuvrer comme les damnés, au point de s'approcher de l'Enfer à la distance d'une coudée, alors ce qui a été écrit pour lui prévaudrait, en sorte qu'il accomplirait les actions des élus et qu'il entrerait (quand même) au paradis " (Bukhârî, 1964, ibid.).

La condition nécessaire pour entrer au paradis et ne pas connaître le feu éternel, et sur laquelle les Maures n'ont aucun doute, consiste à croire en Dieu et en son Prophète Mahomet; la foi en la révélation musulmane a en effet en islam le statut d'œuvre comme le déclare le théologien sunnite Ghazâl1118 (1974 : 3) : "Adam dit : "Ô Seigneur, quelles sont les œuvres des gens destinés au paradis?" Dieu répondit: "Elle sont de trois sortes, la foi en moi, la confiance dans la véracité de mes Envoyés, et l'obéissance à mon Livre, à ses commandements et à ses défenses." " La miséricorde divine ne peut donc s'appliquer à ceux qui ne croient pas en Allah ou à ceux qui lui associent d'autres divinités. Cela explique en partie que la tendance des Maures au prosélytisme soit si forte.

Ils savent en effet qu'un destin infernal attend celui qui ne s'est pas soumis à Dieu, l'attitude de soumission désignant étymologiquement le Musulman. Le Coran décrit en ces termes (XXII, 3-4) la destinée de l'infidèle : "Tel, parmi les hommes discute au sujet de Dieu sans détenir aucune science; il suit tout démon révolté dont il est écrit : "Il égare quiconque le prend pour maître et le dirige vers le châtiment de la flamme brûlante" "(trad. Masson, 1967, t. $2: 408)$.

Toutes les autres fautes, hormis la mécréance et l'associationisme (shirk), peuvent être pardonnées si l'individu s'en repent et s'attache à ne pas récidiver. Ainsi, le Coran promet la peine de l'enfer éternel (IV, 93) à celui qui commet l'homicide volontaire; il s'agit en effet d'une faute

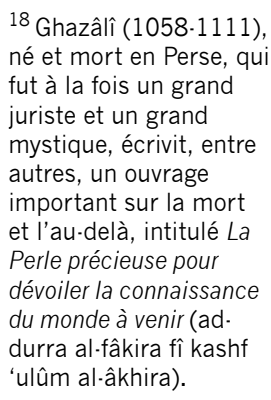

18 Ghazâlî (1058-1111), fut à la fois un grand mystique, écrivit, entre autres, un ouvrage et l'au-delà, intitulé $L a$ Perle précieuse pour du monde à venir (ad. 'ulûm al-âkhira). 
19 L'âne, par exemple, est souvent maltraité dans la société maure, ce qui est aussi le cas dans d'autres sociétés. majeure dans la mesure où non seulement le meurtrier détruit la vie d'autrui mais aussi s'arroge un pouvoir de mort qui n'appartient qu'à Dieu. Cependant, même cette faute peut être pardonnée par le Très-Haut si le coupable s'en repent et ne la renouvelle pas.

Les propos de Ghazâlî (1974 : 78) à ce sujet sont tout à fait éclairants : "Parfois il arrive que le Dieu Très Haut accorde son pardon, au moment où on règle les comptes et les droits des hommes, sauf pour le meurtre avec préméditation, car ce crime n'obtient jamais de pardon, non plus que l'idolâtrie, à moins que les idolâtres ne se convertissent à l'islam, que les meurtriers ne se repentent sincèrement et qu'ils ne retombent plus dans leur péché. Car celui qui tue donne la mort à un être auquel Dieu lui-même avait donné la vie."

En islam, la grâce divine peut s'exercer de façon absolue; c'est ce que suggère l'expression arabe connue dans la société maure: "Par la grâce de Dieu et non par ton mérite " (bi fadlika allahuma lâ bi 'amali). Le Tout-Puissant est libre de prendre ou non en compte les manquements des mortels tout comme leurs œuvres.

Parmi les œuvres, celles qui manifestent cette vertu essentielle qu'est la générosité envers les plus nécessiteux est hautement valorisée et elle est largement pratiquée dans la société maure; l'enfer est, entre autres, le lot de ceux qui auront manqué de porter assistance aux plus démunis, comme l'indique le Coran (LXIX, 33-34) : «Il ne croyait pas en Dieu l'Inaccessible; il n'encourageait personne à nourrir le pauvre "(trad. Masson, 1967, t. 2 : 715).

Les Musulmans se doivent en effet de secourir hommes ou animaux; ainsi, le Prophète déclara qu'une femme respectable qui avait laissé mourir de faim un chat après l'avoir enfermé se retrouverait en enfer tandis qu'une prostituée irait au paradis pour avoir donné de l'eau à un chien qui mourait de soif auprès d'un puits. Mais, dans la société maure, comme dans d'autres sociétés, ce ne sont bien souvent que certaines espèces d'animaux qui font l'objet d'attention tandis que d'autres peuvent être maltraitées ${ }^{19}$. 


\section{CEuvrer pour les morts}

Un hadith montre que le salut de l'âme d'un individu dépend non seulement de lui-même, en particulier de ses œuvres charitables, comme une donation pieuse (waqf) dont les retombées bénéfiques pour les vivants se répéteront de génération en génération, mais également des invocations que ses descendants font en sa faveur : "Lorsque meurent les fils d'Adam, leurs ouvres cessent à l'exception de trois d'entre elles : une aumône dont les effets se perpétuent après sa mort ${ }^{20}$, une science dont on continue de tirer profit, un enfant pieux qui adresse à Dieu des requêtes en leur faveur» (Nawawy, 1991 : 360).

Ainsi, dans la société maure, des invocations, mais aussi des prières, ou encore des aumônes, peuvent être effectuées au nom d'un proche ${ }^{21}$ ou au nom de tous les morts en généra ${ }^{22}$; par exemple, on peut donner de façon effective une offrande à quelqu'un qui en jouira matériellement alors que le bénéfice spirituel en reviendra à celui ou ceux pour qui, en intention, l'aumône a été réalisée.

Il arrive aussi que certaines personnes, dans la société maure comme dans d'autres sociétés musulmanes, accomplissent le cinquième devoir fondamental du Musulman qu'est le pèlerinage (hajj) au nom d'un proche disparu qui n'a pu le faire de son vivant, souvent pour des raisons financières ou de santé. Cet acte, recommandé par le Prophète, influe favorablement sur le salut de l'âme du mort et ne peut être accompli que par quelqu'un qui a déjà réalisé le pèlerinage "pour son propre compte ». Grâce à un tel acte, l'individu cherche à assurer à la fois le salut de l'un de ses proches et le sien propre.

Dans la société maure, c'est souvent à la place de sa mère qu'un homme se rend en pèlerinage, sans doute en raison du fait que le fils est affectivement plus proche de sa mère que de son père mais aussi parce que l'islam attache une grande importance au respect dont elle doit être l'objet. Le statut quasi sacré qui lui est accordé autorise à supposer qu'il est plus méritoire pour un homme d'accomplir le

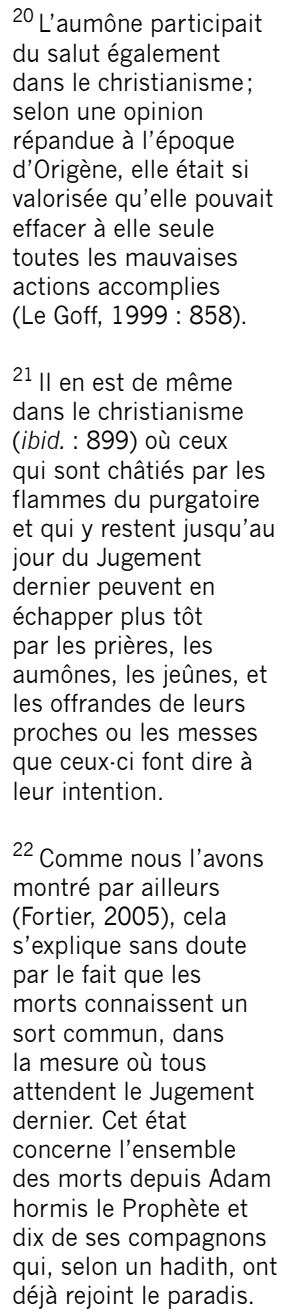

${ }^{22}$ Comme nous l'avons montré par ailleurs (Fortier, 2005), cela s'explique sans doute par le fait que les morts connaissent un sort commun, dans la mesure où tous attendent le Jugement dernier. Cet état concerne l'ensemble des morts depuis Adam hormis le Prophète et dix de ses compagnons qui, selon un hadith, ont déjà rejoint le paradis. 
${ }^{23}$ Nous avons montré par ailleurs (Fortier, 2005) que les morts entendent dans leurs tombeaux, l'ouïe étant le dernier des sens à disparaître. pèlerinage au bénéfice de sa mère qu'à celui de toute autre personne.

Par ailleurs, les œuvres que l'individu accomplit entre l'instant de sa mort et celui de sa résurrection sont prises en compte par Dieu et peuvent contribuer à son salut ou à celui de ses proches. Ainsi, comme le raconte Ghazâlî (1974 : 80), un fils qui aurait manqué de piété filiale durant sa vie peut espérer se rattraper après sa mort et, par cette attitude, infléchir le destin de son père et le sien : " $O ̂$ mon Dieu, j'ai vu que j'étais mis de côté pour l'enfer, sans pouvoir l'éviter, car je me suis mal conduit à l'égard de mon père dans le monde terrestre. Lui aussi se trouve mis de côté pour l'enfer comme moi. Double plutôt ma punition en y ajoutant celle de mon père, et délivre-le de l'enfer". Il dit, et le Dieu Très Haut lui sourit en disant : "Tu as été un mauvais fils dans le monde terrestre, mais tu te montres un bon fils dans celui-ci. Prends ton père par la main et entrez ensemble au paradis." "

\section{Rappeler sa foi en l'islam}

Lors des funérailles, dans la société maure, au moment où les participants ont fini de se recueillir sur le corps du défunt, ils s'éloignent pour laisser la place aux anges qui viennent interroger le mort dans sa tombe. Cette croyance trouve sa source dans un hadith : «Lorsque le croyant a été mis dans son tombeau, que ses amis s'éloignent et retournent chez eux, et alors qu'il entend encore le craquement de leurs sandales ${ }^{23}$, deux anges se rendent auprès de lui, le font mettre sur son séant et lui posent la question suivante : "Que disais-tu de cet homme, Mahomet?" - "Je déclarais, répondra-t-il, qu'il est le serviteur et l'Envoyé de Dieu." Alors, les anges lui diront : "Regarde la place que tu aurais occupée en enfer et celle que Dieu en échange t'a donnée dans le paradis." Et l'homme verra ces deux places. Quant au mécréant ou à l'hypocrite, il répondra à la question posée: "Je ne sais pas; je répétais ce que tout le monde disait." Alors, on dira à cet homme : "Tu n'as rien su, tu n'as donc rien lu?" Et les anges le frapperont une seule 
fois avec un maillet de fer entre les deux oreilles. L'homme poussera un tel cri que tout le voisinage l'entendra, sauf les hommes et les génies " (Bukhârî, 1977 : 430-431).

Les anges qui avaient tout d'abord retiré l'âme de la personne à l'instant de son décès, la réintroduisent dans le corps le temps de l'interrogatoire afin qu'elle puisse répondre à leurs questions. Ces deux anges, Munkar et Nâkîr ${ }^{24}$, ont, selon les Maures, un aspect effroyable: ils ont des dents pointues qui entrent dans la terre et des yeux exorbités qui jettent des flammes; menaçants, ils brandissent des sortes de massue.

La description qu'en fait Ghazâlî (1974 : 20) est à cet égard édifiante: "Ensuite pénètrent auprès du mort les deux questionneurs du tombeau. Ce sont deux anges noirs, qui déchirent la terre avec leurs dents; ils ont de longs cheveux flottants qui traînent sur le sol; leur voix est comme le tonnerre qui gronde avec violence, leurs yeux sont comme l'éclair qui brille, leur souffle est comme le vent impétueux. Chacun d'eux tient à la main une barre de fer si énorme que les hommes et les génies, s'ils unissaient leurs efforts, ne parviendraient pas à la soulever. Si ces deux anges frappaient d'un seul coup de cette barre la montagne la plus gigantesque, ils l'anéantiraient. »

Dans la société maure, on redoute l'interrogatoire promis à celui qui vient de décéder et, comme dans de nombreuses sociétés musulmanes, l'audition est le sens le plus important (Fortier, 1997), on évoque particulièrement le ton tonitruant et terrifiant des anges à ce moment. Seuls les Musulmans qui y auront été préparés trouveront la force de répondre alors que les autres non seulement ne sauront pas quoi dire, mais, apeurés, pourront perdre leur voix. Quant à celui qui hésite à répondre, il sera douloureusement châtié et poussera d'horribles cris de douleur ${ }^{25}$ : "L'homme hésitant sera alors frappé à grands coups de barres de fer, et il poussera des hurlements de douleur que tout l'univers entendra, à l'exception des hommes, car, si les hommes les entendaient, ils perdraient connaissance " (Ghazâlî, 1974 : $402)^{26}$.

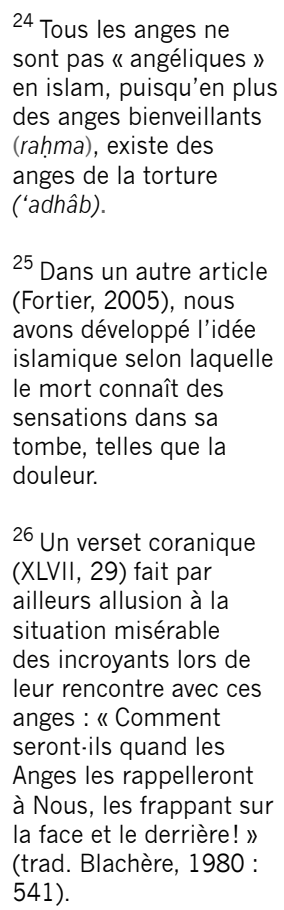


27 À celui qui se demandait comment le mort allait pouvoir dialoguer avec les anges dont il ne connais. sait pas la langue, le syriaque (suryâniyya), le Prophète aurait répondu que ceux qui étaient bénis par Dieu sauraient comment leur répondre.

28 II est par ailleurs impossible qu'un « infidèle » trompe les anges en se faisant passer pour un Musulman puisque, comme le rappelle un verset coranique (XXII,

17), la vérité sera révélée au jour de la résurrection.

${ }^{29}$ Nous avons expliqué ailleurs (Fortier, 2003) pourquoi il nous

semblait plus approprié de rendre le terme de shahâda par « témoi. gnage de la foi » plutôt que par l'expression admise de « profession de foi $»$

${ }^{30}$ Un non-musulman qui prononcerait au seuil de la mort cette formule se verrait aussitôt converti à l'islam et l'ensemble de ses fautes lui seraient du même coup pardon. nées. La conversion apparaît comme une nouvelle naissance puisque l'individu redevient tel qu'il était à sa naissance alors qu'il était porteur de la vérité musulmane.

${ }^{31}$ Nous avons explicité ailleurs (Fortier, 2003) cette nécessité pour les Musulmans de rappeler leur foi en l'islam.
Aussi, dans la société maure, non seulement les individus sont-ils informés de leur vivant de l'interrogatoire qui les attend après leur mort, mais les réponses qu'ils doivent donner leur seront rappelées au moment de leur inhumation afin qu'ils ne manifestent aucune hésitation lorsque les anges les interpelleront sans ménagement. En l'occurrence, à la fin de la prière des funérailles, alors que tous les fidèles se sont retirés, l'imâm reste seul avec le défunt pour lui rappeler ce qu'il devra répondre aux anges quand ceux-ci viendront l'interroger ${ }^{27}$; il lui tient alors ce discours : «Untel, dès qu'ils viennent, ils te demanderont quel est ton dieu, quelle est ta religion, quel est ton prophète, et quel est ton livre. Réponds-leur : mon Dieu est Allah, ma religion est l'islam, mon Prophète est Muhâmmad, et mon Livre est le Coran."

Lors de l'interrogatoire, en effet, le croyant doit affirmer sa foi en Dieu et en son Prophète; comme l'indique un hadith : "Lorsque les deux anges feront s'asseoir le croyant dans sa tombe, il témoignera qu'il n'y a d'autre divinité que Dieu, et que Muhammad est l'Envoyé de Dieu, ce témoignage sera en confirmation des paroles divines " (Bukhârî, 1993 t. $1: 270, \S 688)^{28}$.

D'autre part, il est par ailleurs recommandé que l'individu récite peu avant de mourir « le témoignage de la foi musulmane " (shahâda $)^{29}$ ou que quelqu'un le fasse en son nom s'il en est incapable (Qayrawânî, s.d. : 105), ce que font la plupart des Maures. L'identité religieuse de l'individu est ainsi reconfirmée en fin de vie ${ }^{30}$ exactement de la même manière qu'elle l'avait été à la naissance (Fortier, 1998 : 207 208); car le fait de rappeler, et ce jusqu'à la mort, sa foi en la révélation musulmane est essentiel en islam ${ }^{31}$.

\section{L'épreuve de l'interrogatoire}

Les Maures plaignent celui qui, après l'interrogatoire, se révèlera être un infidèle dans la mesure où déjà dans sa tombe il connaitra les tourments qui l'attendent en enfer; celle-ci s'assombrira, se réchauffera et la terre qui l'entoure viendra se resserrer comme un étau jusqu'à faire éclater ses 
côtes $^{32}$. La description de Ghazâlî (1974 : 24) témoigne de la violence que lui inflige alors les anges : "Quant à l'impie, les deux anges lui disent : "Qui est ton Seigneur?" Il dit : "Je n'en sais rien." Ils lui disent : "Ah! Tu ne sais pas qui il est, tu ne le connais pas!" Alors ils le frappent avec leurs barres de fer jusqu'à ce qu'il soit englouti jusqu'à la septième terre. Puis la terre le rejette de nouveau dans son tombeau, et ils le frappent à sept reprises " $"$.

L'interrogatoire se déroule comme suit pour le croyant qui séjournera en enfer selon une durée plus ou moins longue avant de rejoindre le paradis: "Ils (les anges) l'apostrophent et le forcent à s'asseoir et à s'adosser. Puis ils lui disent: "Qui est ton Seigneur, etc.?", comme la première fois. Il répond sans aucune équivoque: "Dieu est mon Seigneur, Mahomet est mon prophète, le Coran est mon guide, 1'Islâm est ma religion, la Ka'ba est ma Qibla, Abraham est mon père, sa foi est la mienne." Ils lui disent: "Tu as raison." Puis ils agissent envers lui comme la première fois ${ }^{34}$, sauf qu'ils ouvrent une porte de son côté gauche, donnant sur l'enfer. Il peut ainsi contempler les serpents de l'enfer, ses scorpions, ses chaînes et ses carcans, sa chaleur extrême, sa nourriture infernale, sa boisson infecte et tout l'ensemble de ses tortures. Il en est tout tremblant. Alors ils lui disent: "Il ne te sera pas fait de mal. Voici quelle aurait été ta place en enfer, mais Dieu t'en a donné une autre en échange dans le paradis. Dors heureux!" Puis ils referment la porte donnant sur l'enfer, mais ils ouvrent celle qui donne sur le paradis. Le mort ne s'aperçoit pas du passage des mois, des années et des siècles " (Ghazâlî, ibid. : 22).

En revanche, tout individu, dans la société maure, espère faire partie de ceux qui ne connaitront pas les flammes de l'enfer. Dans l'attente du jour de la résurrection, il sera donné au bienheureux un avant-goût du lieu de délices que représente le paradis : l'interrogatoire terminé, sa tombe s'élargira, s'embaumera et deviendra lumineuse et fraîche.

Devant l'assurance émanant des propos de celui qui subit l'interrogatoire, ce qui témoigne de sa confiance en Dieu, les anges lui procureront «luxe, calme et volupté »;

\footnotetext{
32 L'idée de châtiment associée à la sensation de renfermement et de prison se retrouve également dans les conceptions de l'au-delà de l'Égypte pharaonique (Yoyotte, $1961: 68$ ).

${ }^{33}$ Selon le Coran, il existe sept terres, la première étant celle que nous habitons, et la dernière l'enfer. Paral. lèlement, il existe sept cieux, le dernier étant le paradis. Cette croyance se retrouve dans bien d'autres traditions religieuses.

${ }^{34}$ Ghazâlî fait ici référence à ceux qui entreront directement au paradis et qu'il a cités en premier dans l'ordre de présentation de son ouvrage.
} 
comme le décrit Ghazâlî (ibid. : 21) : "Alors ils lui disent : "Qui est ton Seigneur? Quelle est ta religion? Qui est ton prophète? - Quelle est ta Qibla?" L'homme que Dieu a secouru et qu'il a affermi par sa parole immuable leur répond: "Qui est-ce qui vous a donné autorité sur moi? Qui est-ce qui vous a envoyés vers moi?” C'est là la réponse que font les savants vertueux. Alors l'un des anges dit à son compagnon : "Il a raison! Voici, nous l'avons suffisamment maltraité." Puis ils agrandissent le tombeau au-dessus de lui, en lui donnant la forme d'une voûte immense, et ils lui ouvrent de son côté droit une porte qui donne sur le Paradis. Ensuite, ils lui apportent des vêtements de soie et des parfums du paradis, ils lui font parvenir le zéphire et le repos du paradis. Sa conduite vient vers lui, sous la forme de la plus aimable des créatures, pour qu'il converse et s'entretienne avec elle, et le tombeau se remplit de lumière. Le mort ne cesse pas de se réjouir et de s'égayer aussi longtemps que dure le monde terrestre, jusqu'à la venue de l'heure du Jugement, et rien ne lui est plus agréable que de la voir arriver. "

\section{Le poids des œuvres}

Lorsque l'interrogatoire concerne un Musulman préparé à réciter le témoignage de la foi musulmane (shahâda), comme c'est le cas de tout individu en Mauritanie, la balance entre ses œuvres et ses fautes s'avère déterminante pour conditionner sa destinée. Il semble cependant que Dieu, dans sa grande miséricorde, ait tendance à augmenter la valeur des bonnes actions (hasanât) par rapport aux mauvaises (sayyi'ât), et puisse même pardonner ces dernières; c'est ce que montre un hadith (Bukhârî, 1964 : 100, § 40) : "Tout homme qui embrasse l'Islâm et qui pratique un bon Islâm, Dieu lui pardonnera l'ensemble des fautes commises dans le passé par lui. Ultérieurement, la rétribution pour chaque bonne action sera multipliée (en poids) par dix, ou plus, jusqu'à sept cents. La mauvaise action ne sera comptée que pour elle-même, à moins que Dieu ne la laisse impunie." 
Les anges demandent donc au défunt d'inscrire ses bonnes et mauvaises actions sous la forme d'un « livre " que Dieu examinera le jour de la résurrection afin de déterminer le temps que le croyant passera en enfer. Ainsi que le montre un passage de Ghazâlî (ibid. : 20), l'individu décédé utilise son linceul comme papier, et son index mouillé de salive comme plume, à la demande de l'un des anges : " "Ô serviteur de Dieu, tu vas écrire quelle a été ta conduite." Le mort répond : "Je n'ai avec moi ni encrier ni papier." L'ange répond: "Comment donc! Ton linceul, voilà ton papier! Ta salive, voilà ton encre! Ton doigt, voilà ta plume." Puis il lui coupe un morceau de son linceul et le mort se met à écrire, quand bien même il n'aurait pas su écrire pendant sa vie terrestre. Il se souvient de ses bonnes et de ses mauvaises actions comme s'il ne s'agissait que d'un seul jour. Ensuite, l'ange roule ce fragment d'étoffe et le suspend au cou du cadavre. Puis, l'envoyé de Dieu récite le passage du Coran : "Nous avons suspendu au cou de chaque homme sa destinée, à savoir sa conduite" " 35 .

La dialectique paradoxale entre destin scellé par Dieu et prise en considération des œuvres s'applique également le jour ultime de la résurrection. En effet, certains versets du Coran insistent sur le caractère individuel du Jugement, qui est strictement déterminé par les actes de l'individu sans intervention possible d'un tiers, y compris d'un proche: "Le jour où nulle âme n'aura plus pouvoir en faveur d'une autre " (LXXXII 82, 89) et « Le jour où le coupable aimerait se racheter du tourment de ce jour-là, par ses fils, par sa compagne, par son frère, par son clan qui le protège et par tous ceux qui sont sur la terre, pour être sauvé " (LXX 11-14) (Sourdel, 1961 : 184).

Cependant, une histoire édifiante rapportée par Ghazâlî (ibid. : 79-80) témoigne que, le jour même de la résurrection, les morts peuvent influer sur leur destinée, et ce dans un sens ou dans un autre : «Au jour de la résurrection, on amène un homme dont les bonnes et mauvaises actions se font exactement équilibre, et il n'est pas possible de trouver chez lui une seule bonne ouvre qui fasse pencher la balance. Le Dieu Très Haut, ému de compassion, lui

\author{
${ }^{35}$ Le passage du Coran \\ dont il est question \\ est celui-ci : « Nous \\ attachons son destin \\ au cou de chaque \\ homme. Le Jour de \\ la Résurrection, nous \\ lui présentons un livre \\ qu'il trouvera ouvert : \\ Lis ton livre! II suffit \\ aujourd'hui pour rendre \\ compte de toi-même ॥ \\ (XVII, 13-14, trad. \\ Masson, 1967, t. 1 : \\ 342).
}


dit: "Va au milieu des hommes et cherche quelqu'un qui te donne une bonne action, grâce à laquelle je pourrai te faire entrer en paradis." Il s'en va donc et se met à chercher parmi les hommes; mais il ne trouve personne qui veuille lui parler, et tous ceux à qui il adresse une demande lui répondent: "Je crains que ma balance ne soit trop légère; j'ai plus besoin que toi de mes bonnes œuvres." Il est donc sur le point de désespérer, quand un homme lui dit : "Que cherches-tu?" Il répond: "Je cherche une seule bonne action; je suis déjà passé auprès de gens qui en possèdent des milliers, mais qui sont trop avares pour m'en céder une seule." L'autre répond: "J'ai déjà comparu devant le Dieu Très Haut, mais je n'ai trouvé sur mon feuillet qu'une seule bonne action; je ne pense pas qu'elle puisse m'être d'aucune utilité : prends-la donc, je t'en fais présent." Cet homme s'en va en l'emportant, plein de joie et d'allégresse, et le Dieu Très Haut lui dit : "Comment cela va-t-il?" (Or il le sait parfaitement ; loué soit-il!). L'homme lui répond : "Il m'est arrivé telle et telle chose." Puis on appelle son compagnon, celui qui lui a fait don de cette bonne action. Le Dieu Très Haut lui dit : "Ma générosité dépasse encore la tienne. Prends ton frère par la main et entrez tous deux ensemble en paradis.”"

\section{Sur-vies}

La conduite des Maures pouvant déterminer le sort qui les attend dans l'au-delà, on ne peut comprendre nombre de leurs actions sans considérer la dimension eschatologique qui est au cœur de leur vie. La prise en compte de la foi dans la recherche réintroduit alors la question de l'individu et du religieux dans l'analyse des comportements, y compris parmi ceux qui peuvent avoir une portée sociale.

Il en est ainsi par exemple de la pratique de l'hospitalité, bien connue en Mauritanie comme dans d'autres sociétés musulmanes, et dont bénéficient les étrangers de passage; elle est souvent assimilée à une coutume de type culturel qui occulte sa dimension religieuse. Si elle est effectivement accomplie pour autrui dans le but d'en faire 
un allié, ou encore vis-à-vis du voisinage afin d'acquérir un certain prestige, elle est aussi réalisée dans la perspective de gagner son propre salut ou encore comme moyen d'intercéder auprès de Dieu au bénéfice d'un tiers défunt.

Ce cas n'est pas isolé puisque de nombreux domaines relevant de ce qu'on pourrait appeler l'"éthique", si l'on entend par là ce qui concerne le rapport aux autres et à soi-même, sont largement déterminés par des conceptions religieuses. Car, en dépit de la croyance à la toute puissance de la grâce divine en islam, les œuvres n'en sont pas moins reconnues. Ainsi, certains des actes accomplis sur cette terre par un individu peuvent influer sur sa destinée future.

Or les Musulmans qui préparent leur vie dans l'au-delà assurent simultanément celle qui se prolonge ici-bas après leur mort dans le souvenir des vivants; comme le précise un hadith : "Trois choses accompagnent le mort : deux reviennent, une seule reste avec lui. Ce qui l'accompagne, c'est sa famille, sa fortune et ses œuvres; ce qui s'en retourne, c'est sa famille et sa fortune; mais ses œuvres restent " (Bukhârî, 1964 : 93, § 15).

Si une action sociale peut être une action pieuse, l'inverse est également vrai, de sorte que celui qui se conduit comme un bon Musulman gagne les faveurs de Dieu ainsi que celles de ses contemporains. La réputation d'un individu lui survit, aussi constitue-t-elle une motivation supplémentaire pour bien œuvrer.

Ce que la postérité retiendra d'une personne, c'est la manière dont elle a conduit sa vie, ainsi que le suggère un aphorisme maure : «Dans ce monde, la vie est une histoire que l'on relate, fais que la tienne soit belle à raconter" (dunya mrad rwaya wa li dhak izayan rwaytu). De ce point de vue, un individu ayant fait le bien autour de lui ne meurt pas dans la mesure où, comme l'énonce un vers de poésie arabe recueilli en Mauritanie : "N'a pas vécu celui dont les actes ont été blâmables, et n'est pas mort celui qui laisse le souvenir d'un homme de bien" (ma 'âsha man 'âsha madhmûman kasâ'ibihi/wa lam yamut man yakun bi-l-khayri dhikrahu). 


\section{Références bibliographiques}

Bukhârî, M.

1964 L'Authentique tradition musulmane, Trad. de G.H. Bousquet, Paris, Sindbad.

1977 Les Traditions islamiques, t. 1, Trad. de O. Houdas et de W. Marçais, Paris, Adrien et Jean Maisonneuve, 4 tomes.

1993 Le Sommaire du Sahih al-Boukhari, Beyrouth, Dar al-Kutub al-Ilmiyah, 2 tomes.

Carra de Vaux, B.

1975 «Barzakh ", Encyclopédie de l'islam, t. 1, Leiden, Brill et Paris, Maisonneuve, pp. 1103-1104.

Casta, F.J.

1979 «Le sentiment religieux des Corses face à la mort (Approches d'ethnologie religieuse)", Études Corses 12-13, pp. 77-104.

Coran

1967 Trad. de D. Masson, Paris, Gallimard, 2 tomes.

1980 Trad. de R. Blachère, Paris, Maisonneuve et Larose.

Dinet, E. et Ben Ibrahim, S.

s.d. La Vie de Mohammed, s. l., s. éd.

Fortier, C.

1997 «Mémorisation et audition. L'enseignement coranique chez les Maures de Mauritanie ", Islam et Sociétés au Sud du Sahara 11, pp. 85-105.

1998 «Le corps comme mémoire. Du giron maternel à la férule du maitre coranique ", Journal des Africanistes 68 (1-2), pp. 199-223.

2001 «"Le lait, le sperme, le dos. Et le sang?". Représentations physiologiques de la filiation et de la parenté de lait en islam malékite et dans la société maure de Mauritanie ", Cahiers d'Études Africaines 60 (1), pp. 97-138.

2003 «Soumission, pragmatisme et légalisme en islam », Topique, Spiritualités 85, pp. 149-169.

2005 (à paraître) «La mort vivante ou le corps intercesseur ", Revue du Monde Musulman et de la Méditerranée.

Galal, M.

1937 «Des rites funéraires en Égypte actuelle ", Revue des Études Islamiques, pp. 135-285.

Ghazâlî, A.H.M.

1974 La Perle précieuse, Trad. L. Gautier, Amsterdam, Oriental Press.

Ibn Manzûr, M.

s.d. Lisân al-'arab, Beyrouth, Dâr Ṣâdr, 3 tomes (en arabe). 
Laoust, $\mathrm{H}$.

1977 Les Schismes dans l'islam, Paris, Payothèque.

Le Goff, J.

1999 «La naissance du purgatoire » in Un Autre Moyen Âge, Paris, Gallimard, pp. 771-1231.

Molé, M.

1961 «Le jugement des morts dans l'Iran préislamique » in Le Jugement des morts, Paris, Seuil, pp. 145-175.

Nawawy, I.

1991 Les Jardins de la piété. Les Sources de la Tradition islamique, Paris, Alif.

Qayrawânî, A.Z.

s.d. $\quad$ La Risâla. Épître sur les éléments du dogme et de la loi de l'islam selon le rite mâlékite, Trad. de L. Bercher, Alger, H. Pérès.

Richard, Y.

1991 L'Islam chi'ite, Paris, Fayard.

Sharaf, S.S.M.

1987 Le Rappel et l'invocation de Dieu, Kuwait, Dar al-qalam.

Sourdel, D.

1961 «Le jugement des morts dans l'islam » in Le Jugement des morts, Paris, Seuil, pp. 179-205.

Wensinck, A.J. et Mensing, J.P.

1967 Concordances et indices de la Tradition musulmane, t. 6, Leiden, E.J. Brill, 7 tomes (en arabe).

Westermarck, E.

1926 Ritual and Belief in Marroco, London, MacMillan, 2 tomes.

Wigoder, G.

1996 Dictionnaire encyclopédique du judaïsme, Paris, Cerf et Robert Laffont.

Yoyotte, J.

1961 «Le jugement des morts dans l'Égypte ancienne » in Le Jugement des morts, Paris, Seuil, pp. 17-80. 